\title{
The nanoscale control of reactive fluids on geological processes within the solid Earth - EMU Research Excellence Medal Lecture OLIVER PLÜMPER
}

Utrecht University

Presenting Author: o.plumper@uu.nl

Fluid flow through the Earth's crust controls element redistribution to form mineral deposits, the release and sequestration of greenhouse gases, and it facilitates crustal metamorphism. In permeable systems with a well-connected pore space, fluid transport is largely governed by fluid pressure gradients. In impermeable rocks, deformation may induce permeability by creating interconnected heterogeneities, but without these perturbations, fluid transport is limited along grain boundaries or relies on transformation processes that generate fluid pathways. The latter may facilitate large-scale fluid and mass transport in nominally impermeable rocks without largescale tectonic-induced transport pathways. By coupling two- and three-dimensional electron and X-ray microscopic imaging with statistical microstructural descriptors and deep convolutional neural network modelling we investigate the size distribution and formation mechanisms of fluid pathways during pervasive, hydrothermal alteration of igneous feldspathic rocks. We show that feldspar replacement reactions, affecting an area of $>60 \mathrm{~km}^{2}$, are directly linked to the production of nanoscale porosity $(<100$ $\mathrm{nm})$. Consistent with experiments, our results show that interconnected pore space emerges and evolves dynamically as a result of dissolution-precipitation and is thus independent of tectonic forcing and deformation. We find that the pore space is presently disconnected but must have been connected at the time of reaction. Using digital rock volumes, we reconstruct the reaction-induced porosity and apply pore network modelling to compute the transient permeability. Determination of fluid transport coefficients through feldspar nanopores derived from non-equilibrium molecular dynamics (MD) simulations coupled to continuum-scale fluid mechanics suggests that fluid and mass transport can be self-generated through the emergence of electrokinetic transport processes and may not rely on fluid pressure gradients. We further use MD simulations to investigate the effect of nanoconfinement on fundamental fluid properties, e.g., the dielectric constant. Results suggests that the dielectric properties of water deviate strongly compared to bulk water when confined in nanopores under geo-relevant conditions. This may have dramatic consequences for mineral solubilities and thus mass transport in the Earth's lithosphere, not only in igneous feldspathic rocks. 